\title{
LA ADAPTACIÓN DE LA NORMATIVIDAD MEXICANA EN LA ENSEÑANZA DE LAS ASIGNATURAS DE CONTENIDO AMBIENTAL EN LA DIVISIÓN ACADÉMICA DE CIENCIAS SOCIALES Y HUMANIDADES DE LA UNIVERSIDAD JUÁREZ AUTÓNOMA DE TABASCO, MÉXICO
}

\author{
CARLOS RAFAEL GUAJARDO GOMEZ \\ Maestro en Derecho Civil, Universidad Juárez Autónoma de Tabasco. México (2005) \\ Doctorando en Derecho, Universidad Juárez Autónoma de Tabasco. México \\ Profesor de tiempo completo en la Licenciatura en Derecho de la División Académica de \\ Ciencias Sociales y Humanidades de la Universidad Juárez Autónoma de Tabasco \\ guajardo47@hotmail.com
}

MARTHA SALGADO MIRANDA

Egresada de la Universidad Juárez Autónoma de Tabasco con la investigación titulada

“La Formación de Profesores en el Ámbito Académico de la Educación Superior. Un Estudio de Caso de la Universidad Juárez Autónoma de Tabasco.

Actualmente es Profesora-Investigadora de la División Académica de Ciencias Sociales y Humanidades e imparte cátedras de Metodología de la Investigación y Seminario de Tesis

AREANNA HERNÁNDEZ JIMÉNEZ

\begin{abstract}
Maestría en Derecho con especialidad en Derecho Constitucional de la Universidad Juárez Autónoma de Tabasco con la Tesis “La Sistematización Jurídica de la Protección al Medio Ambiente en el Estado de Tabasco". con mención honorífca.

arihj80@hotmail.com
\end{abstract}

\section{Cómo citar este artículo:}

Guajardo, C. \& Miranda, M. (2011). La adaptación de la normatividad mexicana en la enseñanza de las asignaturas de contenido ambiental en la División Académica de Ciencias Sociales y Humanidades de la Universidad Juárez Autónoma de Tabasco, México. Espiral, Revista de Docencia e Investigación. 1, (1), 119 - 128

\section{Resumen:}

En el presente artículo se realiza un estudio sobre la adecuación y adaptación de la normatividad mexicana en la enseñanza de las asignaturas Cultura Ambiental, Ambiente y sociedad, Desarrollo Sustentable y Derecho Ambiental, en la División Académica de Ciencias Sociales y Humanidades de la Universidad Juárez Autónoma de Tabasco, México. El diseño curricular planteado en los planes y programas de estudio en la Universidad, están elaborados por competencias, buscan acercar al estudiante a la sistematización y ordenamiento del conocimiento científco a través de las asignaturas: Cultura Ambiental, Ambiente y Sociedad, Desarrollo Sustentable y Derecho Ambiental. De acuerdo al campo científco de cada una de ellas en el dominio de la ejecución del valor teórico y los saberes transversales, se expone la construcción y valoración del conocimiento del estudiante hacia una nueva valoración del medio ambiente.

Palabras clave: ambiente, enseñanza-aprendizaje, educación, normatividad, preservación.

\begin{abstract}
:
This article is a study on the adequacy and adaptation of Mexican standards in teaching environmental subjects Culture, Environment and Society, Sustainable Development and Environmental Law in the Academic Division of Social Sciences and Humanities Universidad Juarez Autónoma de Tabasco, Mexi-
\end{abstract}


co. The proposed curriculum plans and programs of study at the University, are produced by competence, seek to bring the student to the systematic and scientifc knowledge management across the subject: Environmental Culture, Environment and Society, Sustainable Development and Environmental Law. According to the scientifc feld of each domain in the implementation of the theoretical value and knowledge cross, we illustrate the construction and assessment of student knowledge was a new appreciation of the environment.

Key words: environment, teaching-learning, education, regulations, preservation.

\section{Introducción}

Para la realización del artículo se utilizó la investigación cualitativa y cuantitativa.

En la investigación cualitativa se abarca el desarrollo metodológico a través del análisis descriptivo de las teorías y disciplinas de las ciencias sociales. En la cuantitativa se explora el objeto de estudio una muestra representativa del $10 \%$ de la matrícula estudiantil, correspondiente al ciclo escolar 2008-2010 en la División Académica de Ciencias Sociales y Humanidades de la Universidad Juárez Autónoma de Tabasco. Con la fnalidad de conocer en este centro de estudios la aplicación de las asignaturas de educación ambiental.

Se tomó como elemento metodológico los fundamentos jurídicos de la normatividad para la investigación y educación ecológicas en México, instrumentos de las políticas públicas de fomento a la conciencia ambiental y al desarrollo sustentable. Así como también el proceso enseñanza-aprendizaje de las asignaturas de contenido ambiental en la División Académica de Ciencias Sociales y Humanidades de la Universidad Juárez Autónoma de Tabasco.

Se describe además, las características de los elementos metodológicos al abordar las teorías del desarrollo sustentable, de la educación ambiental, del derecho ambiental (Gutiérrez, 2007) de confictos socioeconómicos, de la comunicación social y el constructivismo. Es pertinente señalar que las Ciencias Sociales permiten el acercamiento a la práctica de la enseñanza de las asignaturas: Cultura ambiental, Ambiente y sociedad, Desarrollo sustentable y Derecho ambiental.

A lo anterior se agrega la práctica de la enseñanza desde el campo de la investigación en la Educación Superior en México, a través de los métodos científcos utilizados por el profesor que imparte las asignaturas antes mencionadas, con el objetivo de proporcionarle al estudiante las herramientas necesarias para la comprensión de los contenidos y que éstos propician el interés por la dimensión hombre-naturaleza.

En la descripción metodológica se emplearon como variables de análisis, los siguientes enunciados:

- El desarrollo de la educación ambiental en México: políticas públicas ambientales.

- La investigación científca y tecnológica para el desarrollo de la cultura ambiental.

- La normatividad ambiental mexicana en la educación superior.

El artículo enfatiza en el desarrollo de la normatividad del sistema jurídico mexicano en la educación ambiental, por ser la que determina los factores de formación académica en los estudiantes de educación superior, con la fnalidad de crear en ellos una conciencia de protección y conservación del medio ambiente.

Por ello, con la investigación se buscó determinar los procesos de enseñanza-aprendizaje de las asignaturas que se imparten 
en la División Académica de Ciencias Sociales y Humanidades. Dentro de los planes y programas de estudios de las Licenciaturas en Derecho, Historia, Sociología, se contemplan asignaturas cuyo contenido abarca el fomento sustentable del medio ambiente y su regulación a través de las normas protectoras de éste. Así, se busca mostrar la importancia que tiene para nuestro país y específcamente para la División Académica de Ciencias Sociales y Humanidades, proveer al estudiante de la cultura conservacionista.

\section{Desarrollo de la Cultura Ambiental en México}

\subsection{Antecedentes de la}

\section{Educación Ambiental en México}

En la década de los setenta, con el auge de diversos movimientos sociales, se exhibió el daño y deterioro ambiental. (Carmona Lara; 2001: 30). La crisis ambiental demandaba una respuesta social por parte de los Estados, por ello las naciones se reunieron con la fnalidad de llegar a concertaciones y aplicar en conjunto, políticas de gobierno, que aportaran una solución a la problemática ambiental-social. La educación ambiental, surge en los años 70, como una de las respuestas a esta problemática. Con ella se busca lograr un cambio de cultura y de mentalidad con respecto al comportamiento hacia el medio ambiente.

Esta directriz se discutió a nivel mundial en la primera gran Conferencia de las Naciones Unidas Sobre el Medio Ambiente
Humano, celebrada el 5 y 6 de junio de 1972, en Estocolmo, Suecia, que produjo la Declaración de Estocolmo, se emitió el diagnóstico de los problemas ambientales más acuciantes, en un texto de 26 principios, que sentaron las bases del comportamiento que los pueblos deben observar en la preservación del medio ambiente (López y Ferro; 2006: 54-55) y en educar para su realización al señalar en su principio 19:

"Es indispensable una labor de educación en cuestiones ambientales, dirigida tanto a las generaciones jóvenes como a los adultos y que preste la debida atención al sector de población menos privilegiado, para ensanchar las bases de una opinión pública bien informada, y de una conducta de los individuos, de las empresas y de las colectividades inspirada en el sentido de su responsabilidad en cuanto a la protección y mejoramiento del medio ambiente en toda su dimensión humana".

En México, con la creación de la Secretaría de Desarrollo Urbano y Ecología (SEDUE) se institucionalizó la educación ambiental en 1982. A partir de 1985 el Centro de Estudios sobre la Universidad (CESU) de la Universidad Nacional Autónoma de México (UNAM), elaboró un estudio para la Secretaría de Educación Pública (SEP) sobre la necesidad de fortalecer la dimensión ambiental en los diferentes niveles y modalidades educativos. En marzo de 1992 se celebró la Primera Reunión Nacional de Educadores Ambientales; de donde surgió la primera estrategia nacional en el rubro. La década de 
los noventa se distingue por la generación de diversos grupos y redes de ambientalistas, ecologistas, educadores y gubernamentales, quienes se congregaron en eventos académicos para la capacitación e intercambio de experiencias. En el 2005 se llevó a cabo el Foro Nacional: 10 años para cambiar al mundo celebrado en Aguascalientes, donde se suscribió el compromiso nacional entre las empresas, gobierno, universidades y organismos sociales, para trabajar en la construcción colectiva de la educación ambiental para el país. (Bravo y Reyes; 2008: 15-18).

\subsection{Políticas públicas ambientales}

En México le corresponde a la Secretaría de Educación Pública (SEP), con base en la estructura gubernamental delegada por el Poder Ejecutivo, la toma de decisiones, para reconocer una política jerárquicamente operativa. (Ley Orgánica de la Administración Pública Federal, Diario Ofcial de la Federación, 2009) Donde existe un aparato administrativo que expresa su compromiso y actúa en la escala nacional para aterrizar hacia lo local.

El marco legal del Sistema Educativo Nacional se fundamenta en el artículo tercero de la Constitución Federal, del cual se deriva su ley reglamentaria, la Ley General de Educación. Las políticas educativas se encuentran delineadas en el artículo 7 , fracción XI de esta ley, donde señala los fnes que persigue el Estado, sus organismos descentralizados y los particulares al impartir educación en el área ambiental:

"Inculcar los conceptos y principios fundamentales de la ciencia ambiental, el desarrollo sustentable, la prevención del cambio climático, así como de la valoración de la protección y conservación del medio ambiente como elementos esenciales para el desenvolvimiento armónico e integral del individuo y la sociedad.
También se proporcionarán los elementos básicos de protección civil, mitigación y adaptación ante los efectos que representa el cambio climático y otros fenómenos naturales.

Esto ha propiciado que en las políticas públicas del medio ambiente, se legisle la educación ambiental vista como un instrumento primordial para su consecución. Defnida en la Ley General del Equilibrio Ecológico y la Protección al Ambiente (LGEEPA), en su artículo 3, fracción XXXVII de la siguiente manera:

"Proceso de formación dirigido a toda la sociedad, tanto en el ámbito escolar como en el ámbito extraescolar, para facilitar la percepción integrada del ambiente a fn de lograr conductas más racionales a favor del desarrollo social y del ambiente. La educación ambiental comprende la asimilación de conocimientos, la formación de valores, el desarrollo de competencias y conductas con el propósito de garantizar la preservación de la vida"

\subsection{Investigaciones científcas y tecno- lógicas para el desarrollo de la cultura ambiental}

Una tendencia más de carácter internacional para el fomento de la cultura proteccionista del ambiente, es la investigación científca y tecnológica consagrada en la Declaración de Estocolmo en el Principio 20, al enunciar:

Se deben fomentar en todos los países en desarrollo, la investigación y el desarrollo científcos referentes a los problemas ambientales, tanto nacionales como multinacionales. A este respecto, el libre intercambio de información científca actualizada y de experiencias sobre la transferencia debe ser objeto de apoyo y asistencia, a fn de facilitar la solución de los 
problemas ambientales; las tecnologías ambientales deben ponerse a disposición de los países en desarrollo en condiciones que favorezcan su amplia difusión sin que constituyan una carga económica excesiva para esos países.

Los elementos de la educación ambiental están constituidos en un primer momento por la información de qué es o qué constituye el medio ambiente, con cada uno de sus componentes como son los elementos físicos, químicos, biológicos y humanos que lo constituyen, para lograr en el educando la conciencia de que los seres humanos son parte integrante del medio ambiente y no un factor ajeno a él. (Smith, 1997)

Uno de los objetivos generales es lograr que los individuos y las colectividades comprendan la naturaleza compleja del medio ambiente natural y del creado por el hombre, la adquisición de conocimientos, valores, comportamientos y habilidades prácticas para participar responsable y efcazmente en la prevención y solución de los problemas ambientales y en la gestión de la cuestión de la calidad del medio ambiente. (Terrón; 2000:6)

En México esta directriz forma parte de las políticas públicas ambientales, preceptuado en la LGEEPA, la cual contempla como instrumentos de la política ambiental, la Investigación y Educación Ecológicas, artículo 39, fracciones I, III y IV expresan:

I. Las autoridades competentes promoverán la incorporación de contenidos ecológicos conocimientos, valores $\mathrm{y}$ competencias, en los diversos ciclos educativos, especialmente en el nivel básico, así como en la formación cultural de la niñez y la juventud.

III. La Secretaría de Medio Ambiente y Recursos Naturales (SEMARNAT), con la participación de la Secretaría de
Educación Pública (SEP), promoverá que las instituciones de Educación Superior y los organismos dedicados a la investigación científca y tecnológica, desarrollen planes y programas para la formación de especialistas en la materia en todo el territorio nacional y para la investigación de las causas y efectos de los fenómenos ambientales.

IV. La Secretaría mediante diversas acciones promoverá la generación de conocimientos estratégicos acerca de la naturaleza, la interacción entre los elementos de los ecosistemas, incluido el ser humano, la evolución y transformación de los mismos, a fn de contar con información para la elaboración de programas que fomenten la prevención restauración, conservación y protección del ambiente. (LGEEPA, última reforma, 28-01-2011b)

Muestra clara de la aplicación de este precepto es la inclusión de las asignaturas: Cultura ambiental, Ambiente y Sociedad, Desarrollo Sustentable y Derecho Ambiental, en los planes de estudio de las licenciaturas en Derecho, Historia y Sociología que se imparten en la División Académica de Ciencias Sociales y Humanidades (DACSYH) de la Universidad Juárez Autónoma de Tabasco (UJAT)

Científcamente se crea una conciencia ecológica conservacionista, presente aun cuando la ciencia y la tecnología propician deterioros ambientales, son éstas las que plantean bases científcas para proteger al medio ambiente y esto se convierte en educación en la medida que los paradigmas sociales asumen la responsabilidad y a través de los gobiernos se trabaja en función de proteger al medio ambiente. La UJAT en sus planes y programas de estudio se enfoca en el proceso de enseñanza-aprendizaje a trabajar en las políticas educativas a nivel superior. 


\section{La Normatividad ambiental mexicana en la educación superior}

\section{La normatividad mexicana aplicable a la educación ambiental}

La normatividad mexicana aplicable a la educación ambiental en el nivel superior, recae en la Constitución Política de los Estados Unidos Mexicanos, primer y máximo ordenamiento del sistema jurídico nacional. Los artículos constitucionales que fundamentan principios ambientales son: 2 apartados $\mathrm{A}$ y B, fracción V y VII; 3, 4 párrafo cuarto, 25 párrafo primero, 27 párrafo tercero, 73 fracción XXIX-g, 115 fracción V inciso g y 122 base primera, fracción $\mathrm{V}$, inciso $\mathrm{j}$.

De su contenido se desprende en el orden antes mencionado: el derecho de los pueblos indígenas a conservar y preservar su hábitat; la educación ambiental; el derecho de todos los habitantes a gozar de un ambiente sano; el desarrollo nacional integral y sustentable; el aprovechamiento y uso adecuado de los elementos naturales que preservan y restauran el equilibrio ecológico; la concurrencia de facultades entre la federación, los estados y municipios en materia de protección al ambiente y de preservación y restauración del equilibrio ecológico; la administración de los municipios en las zonas de reserva ecológica; la facultad del Distrito Federal (capital del país) de legislar en materia de preservación del medio ambiente y protección ecológica. (Constitución política de los Estados Unidos Mexicanos. 1917. Diario Ofcial de la Federación última reforma 17-08-2011).

En cuanto a la legislación resultado de los mandatos constitucionales, además de la LGEEPA en la que se basa la política ambiental del país, se encuentran las siguientes: Ley de Sanidad Fitopecuaria de los Estados Unidos Mexicanos (1974); Ley General de Bienes Nacionales (1982); Ley General de Salud (1984), Ley Federal del Mar (1986),
Ley de Aguas Nacionales (1992), Ley Minera (1992). Ley Agraria (1992), Ley Federal sobre Metrología y Normalización (1992), Ley Federal de Sanidad Animal (1993), Ley General de Asentamientos Humanos (1993), Ley Federal de Sanidad Vegetal (1994), Ley Federal de Variedades Vegetales (1996), Ley General de Desarrollo Forestal Sustentable (1997), Ley General de Vida Silvestre (2000), Ley General para la Prevención y Gestión Integral de los Residuos (2003), Ley de Bioseguridad de Organismos Genéticamente Modifcados (2005), Ley General de Pesca y Acuacultura Sustentables (2007), Ley para el aprovechamiento de energías renovables y el fnanciamiento de la transición energética (2008) y la Ley de Promoción y Desarrollo de los Bioenergéticos (2008). (INE, 2010). Código Civil Federal; Código Penal Federal y las Normas Ofciales Mexicanas (NOM) en materia de protección al ambiente y al equilibrio ecológico

Puede apreciarse que el catálogo de normas es amplio y variado, además se cuenta con el principio de soberanía de los Estados de la Unión en todo lo que compete a su régimen interior y así resulta que el tema ambiental, al no estar reservado por la Constitución de manera exclusiva a la Federación, puede ser regulado de manera concurrente por las entidades federativas en lo que corresponda a su régimen interno, por lo que cada estado tiene su propia normatividad ambiental.

De igual manera, los tratados internacionales son de importancia por ser normas de derecho vinculante en el ámbito internacional y en el ámbito nacional tienen la categoría de ley suprema, con base en al artículo 133 de la Constitución Federal. El Estado Mexicano ha suscrito un importante número de tratados internacionales, 12 bilaterales y 47 multilaterales (Simental; 2010: 311-316) reguladores de diversas materias ambientales, insertándolos en su legislación nacional. 
Esta legislación se refeja en los contenidos temáticos de los programas de estudio de las asignaturas en comento en la DACSYH-UJAT. En la revisión al contenido de los programas se encuentran los siguientes componentes: la evolución del Derecho Económico al nacimiento del Derecho de Protección al Ambiente, los tratados y principios internacionales para la protección del ambiente, el marco jurídico y las autoridades ambientales en México.

Esto permite la construcción de los conocimientos para la comprensión del entorno en los ámbitos regional, nacional e internacional, el sentido ético y social de las posturas flosófcas y epistemológicas acerca del ambiente, la relación hombre-naturaleza, el medio ambiente y desarrollo, los modelos de desarrollo, el desarrollo sustentable, las políticas ambientales, la visión global de los problemas ambientales, así como los elementos estratégicos para el desarrollo de la educación ambiental en México.

Comprendidos desde una perspectiva global, el neoliberalismo, la geopolítica mundial, el desastre ecológico, pobreza extrema mundial, los límites del crecimiento económico, el desarrollo sustentable como una solución a la crisis de la sociedad industrial, lo que permite la participación social y los movimientos sociales ambientalistas, los cuales buscan incluir en los discursos la inclusión de la perspectiva de género, medio ambiente y desarrollo sustentable en las políticas de desarrollo, el ecofeminismo como el género del ambiente, la cultura y el consumo sustentable, la educación ambiental y las políticas públicas.

Con base en la descripción anterior, se interpreta que la legislación sirve a la sociedad para que se vinculen en la educación los preceptos legales $\mathrm{y}$, de esta manera, la educación superior específcamente la
UJAT promueva el conocimiento científco en las ciencias ambientales.

3. La enseñanza de las asignaturas: Cultura ambiental, Ambiente y sociedad, Desarrollo sustentable y Derecho ambiental

El proceso enseñanza-aprendizaje de las asignaturas de contenido ambiental en la División Académica de Ciencias Sociales y Humanidades de la Universidad Juárez Autónoma de Tabasco

Como se ha comentado en capítulos anteriores, acerca de los antecedentes de la educación ambiental, las políticas públicas y la legislación aplicable, para este capítulo es necesario señalar que el plan de estudios de la UJAT basado en el enfoque por competencias propone la realización de una educación, para la conservación del medio ambiente.

La enseñanza-aprendizaje se defne como el proceso mediante el cual se comunican o transmiten conocimientos especiales o generales sobre una materia y, por el cual, una persona es capacitada para dar una solución a situaciones; tal mecanismo va desde la adquisición de datos hasta la forma más compleja de recopilar y organizar la información. (Edel, 2004).

Los paradigmas sociales, económicos, socio-políticos que plantean la necesidad de conformar esos saberes distancian el momento de su estudio para ser aplicados a nivel superior.

Desde esta perspectiva enfatiza la necesidad de atender en los programas de estudio los contenidos de aprendizaje en torno a la exigencia de percibir el deterioro ambiental, que genere en el estudiante los conocimien- 
tos para hacerlo consciente de su realidad y de su potencial de participar en la toma de decisiones y realizar acciones positivas y de cambio.

\section{Metodología}

Para acercarnos al objeto de estudio y corroborar la información se elaboró un estudio de campo que permitió conocer cómo se desarrolla dentro de las aulas el contenido educativo de las asignaturas ambientales. Para ello se aplicó un cuestionario de trece preguntas a una muestra representativa del $10 \%$ de la matrícula estudiantil, correspondiente al periodo escolar 2008-2010, en la DACSYH-UJAT. De acuerdo al enfoque del estudio descriptivo correlacional se explican los resultados obtenidos en las encuestas:

1. El $100 \%$ de los encuestados responde tener conocimiento sobre la educación ambiental en México, antes de haber cursado la materia de Cultura ambiental en la universidad. De lo anterior se deduce que el trabajo educativo en esta materia servirá para reforzar los conocimientos adquiridos en otros niveles educativos. La información que proporciona la encuesta, da las bases para considerar que es correcto encauzar a los estudiantes a la realización de investigaciones científcas, de manera interdisciplinaria, asociadas con las diversas disciplinas de su trayectoria académica.

2. El 75\% consideró que las políticas públicas ambientales son de carácter político, al apreciar que son los gobiernos y sus instituciones quienes impulsan el cuidado y protección del ambiente. Esiendo tarea de los profesores de las materias de contenido ambiental reforzar el conocimiento y reorientarlo hacia la toma de conciencia de que sí bien los gobiernos tienen la responsabilidad de programar, desarrollar y ejecutar la política ambiental, la sociedad es igualmente corresponsable. Ahora bien el restante $25 \%$ de los encuestados estima que las políticas públicas son de carácter informativo, por tal razón los educadores deberán promover la adecuada formación de los estudiantes de educación superior, a fn de que tengan un conocimiento integral del signifcado de las políticas públicas ambientales.

3. Cuando se les cuestionó sobre qué factores intervienen en la educación ambiental, el 75\% argumenta que el factor sociocultural es el predominante en la percepción del cuidado del entorno. Asimismo el otro 25\% afrmó que el factor político marca la tendencia en la protección de los bienes naturales.

4. Continuando con el cuestionamiento el 75\% afrma conocer leyes que contemplan la educación ambiental y su aplicación en todos los niveles educativos. Por otra parte un $25 \%$ desconoce la existencia de una legislación que señale la obligatoriedad de impartir la en los diversos niveles educativos.

5. Sobre la formación educativa ambiental, se les preguntó cuál es la norma jurídica que incluye la educación ambiental como proceso de formación, se obtuvo que un $100 \%$ dice conocer con exactitud el artículo 39 de la LGEEPA, el precepto normativo de la educación ambiental en nuestro país. De acuerdo a los resultados se observó que el trabajo realizado por los profesores investigadores que imparten las asignaturas de contenido educativo ambiental han logrado cumplir con los objetivos del proceso de enseñanza aprendizaje que proponen los planes de estudios de las licenciaturas en Historia, Sociología y Derecho. 
6. Un cuestionamiento más que se formuló es acerca de si las normas ambientales fomentan adecuadamente la educación ambiental, un 50\% respondió en sentido positivo, a su parecer cumplen con fomentar la conservación del medio ambiente. Un 25\% aprecia que la educación superior debe fomentar la cultura conservacionista del entorno. El 25\% restante dice que es debido a la educación ambiental que se protegen los recursos naturales.

7. Al preguntarles si consideran adecuadas las asignaturas de contenido ambiental que se imparten en la universidad, el 100\% responde que sí, porque se adquiere conocimientos conservacionistas y se logra una mayor comprensión de la responsabilidad de proteger el medio ambiente.

8. De acuerdo al interrogante que se plantea si las asignaturas de contenido ambiental en los programas de las licenciaturas de la División Académica, son de carácter formativo integral hacia la sustentabilidad, el 100\% contestó afrmativamente.

9. Al pedirles su opinión respecto del perfl de los profesores que imparten las materias ambientales en las tres licenciaturas de la División, el 75\% expresa que los profesores investigadores cuentan con el perfl adecuado en la temática ambiental y el $25 \%$ sobrante contestó que sus profesores se actualizan constantemente en el área.

10. También se indagó si conocen que en su División Académica se realicen investigaciones sobre el tópico ambiental, encontrándose que un 100\% sabe que se realizan y que les han sido de utilidad en su formación académica.
11. De igual manera se observó que en un $100 \%$ los estudiantes han realizado y participado en proyectos de investigación científca en el tema ambiental.

12. Otro interrogante, es si perciben la aplicación del desarrollo sustentable como modelo económico y social en armonía con la naturaleza, el 75\% respondió que en México, este modelo se aplica en el desarrollo nacional para la protección del medio ambiente y el equilibrio ecológico.

13. Para fnalizar se preguntó cuáles son los benefcios que las asignaturas ambientales han dejado en su formación, el 100\% argumentó que gracias a éstas son conscientes de la responsabilidad que tienen todos los sectores de una sociedad de participar y contribuir en el cuidado de la naturaleza, sus elementos y recursos naturales.

\section{Conclusión}

El soporte teórico del constructivismo radica en vincular la concepción del aprendizaje como factor sociocultural predominante en la percepción del cuidado del entorno. Las referencias previas de los estudiantes respecto al entorno, se generan en la interacción social en la relación sujeto-medio ambiente, estos resultados los podemos aplicar en el desarrollo de los procesos educativos de las asignaturas ambientales en educación superior.

El concepto de aprendizaje signifcativo, surge cuando el estudiante como los niveles constructor de su propio conocimiento, relaciona lo aprehendido y le da una aplicación, en este contexto el estudiante es constructor de su propio conocimiento en un sentido formativo sobre la temática ambiental. Al unir conocimientos previos con conocimientos nuevos el estudiante adquiere estructuras 
cada vez más complejas del entorno social, es consciente de su realidad y le da una signifcación valorativa.

El tema de investigación abordado despierta gran interés por su pertinencia al contexto educativo que se logra a través de vincular la normatividad a la educación ambiental. Tema, por demás, complejo al intervenir factores políticos, económicos, culturales y sociales en la educación superior. Abordar los fenómenos de la conservación del medio ambiente es un gran desafío, por el impacto en el deterioro de la naturaleza. La integración de los procesos de enseñanza-aprendizaje, crean conciencia en el estudiante, pero es la búsqueda hacia nuevas perspectivas en el análisis que abren el debate acerca de que la sola concientización es insufciente, por ello la realización de acciones colectivas que propicien exigir nuevas alternativas o modelos de situaciones de cambio para lograr la disminución de la pérdida del capital natural.

Los esfuerzos de la Universidad Juárez Autónoma de Tabasco han impactado en la educación de generaciones propositivas, al adaptar la normatividad mexicana en la enseñanza de los contenidos de carácter ambiental. Sin embargo, los desafíos son muchos y las problemáticas complejas, sobre ello se tiene que actuar en la toma de decisiones.

\section{Referencias bibliográfcas}

Bonnefous, Edouard, (1973). ¿El Hombre o la Naturaleza? México: Fondo de Cultura Económica.

Carmona, María, (2001). Derechos en Relación con el Medio Ambiente. México: Cámara de Diputados LVIII Legislatura.

Conferencia de las Naciones Unidas sobre el Medio Humano. Declaración de Estocolmo, Junio de 1972. Disponible en: http://www.oarsoaldea. net/agenda21/es/node/6 . (Recuperado 20 de septiembre de 2011)
Constitución Política de los Estados Unidos Mexicanos, Diario Ofcial de la Federación, última reforma, 13 de abril de 2011. Disponible en: http:/ / www.diputados.gob.mx/ LeyesBiblio/. (Recuperado 28 de septiembre de 2011)

Edel, R. (2004). El concepto de enseñanza aprendizaje. Disponible en: http://www.redcientifca. com/doc/doc200402170600.html (Recuperado 28 de septiembre de 2011)

H. Congreso de la Unión, Cámara de Diputados LXI legislatura, Ley General deEducación, Diario Ofcial de la Federación, última reforma publicada, 28 de enero de 2011. Disponible en http:/ / www. diputados.gob.mx/LeyesBiblio/. (Recuperado 28 de septiembre de 2011)

H. Congreso de la Unión, Cámara de Diputados LXI legislatura, Ley General del Equilibrio Ecológico y la Protección al Ambiente, Diario Ofcial de la Federación, última reforma publicada, 28 de enero de 2011.

Instituto Nacional de Ecología (INE), (2010). Disponible en http:/ / www2.ine.gob.mx/ publicaciones/ libros/614/documentacion.pdf/20. (Recuperado 26 de septiembre de 2011)

Juste, J. (1999). Derecho Internacional Ambiental. Madrid: McGraw Hill.

López, P. y Ferro, A. (2006). Derecho Ambiental. México: Iure Editores

Quintana, J. (2005). Derecho Ambiental Mexicano, Lineamientos Generales. México: Porrúa.

Reyes, F. y Bravo, T. (2008). Educación Ambiental para la sustentabilidad eMéxico. Aproximaciones conceptuales, metodológicas y prácticas. México: Universidad de Ciencias y Artes de Chiapas.

Simental, V., (2010). Derecho Ambiental. México: Limusa.

Smith, N.J., (1997). ¿Qué es educación ambiental? EstadosUnidos: University of Illinois Cooperative Extension Service.

Terrón, E. (2000) La educación ambiental ante los desafíos del siglo XXI, en: Revista de la Academia Mexicana de Profesores de Ciencias Naturales A. C. México: Universidad Pedagógica Nacional. 
